\title{
$\left({ }^{3} \mathrm{He}, \mathrm{t}\right)$ Charge-Exchange Reactions at $\mathrm{E}\left({ }^{3} \mathrm{He}\right)=450 \mathrm{MeV}, \theta=0^{\circ}$
}

H. Akimune ${ }^{1}$, I.Daito ${ }^{2}$, Y. Fujita ${ }^{3}$, M. Fujiwara ${ }^{2}$, M.B. Greenfield ${ }^{4}$, M.N. Harakeh ${ }^{5}$, T. Inomata ${ }^{6}$, J. Jänecke ${ }^{7}$, K. Katori ${ }^{6}$, S. Nakayama ${ }^{8}$, H. Sakai ${ }^{9}$, Y. Sakemi ${ }^{2}$, M. Tanaka ${ }^{10}$, and M. Yosoi ${ }^{1}$

${ }^{1}$ Department of Physics, Kyoto University, Kyoto 606, Japan

${ }^{2}$ Research Center for Nuclear Physics, Osaka University, Mihogaoka 10-1, Ibaraki, Osaka 567 Japan

${ }^{3}$ College of General Education, Osaka University, Toyonaka, Osaka 560, Japan

${ }^{4}$ Natural Sciences Division, International Christian University, Mitaka, Tokyo 181, Japan

${ }^{5}$ Kernfysisch Versneller Instituut, Zernikelaan 25, 9747 AA Groningen, The Netherlands

${ }^{6}$ Department of Physics, Osaka University, Toyonaka, Osaka 560, Japan

${ }^{7}$ Department of Physics, University of Michigan, Ann Arbor, MI 48109-1120, USA

${ }^{8}$ College of General Education, Tokushima University, Tokushima, Japan

${ }^{9}$ Department of Physics, University of Tokyo, Bunkyoku, Tokyo, 113 Japan

${ }^{10}$ Kobe Tokiwa Jr. College, Nagata, Kobe 653, Japan

\begin{abstract}
Spin-isospin excitalions in nuclei have been studied by means of the $\left({ }^{3} \mathrm{He}, \mathrm{l}\right)$ reactions at $450 \mathrm{MeV}$ on the targets ${ }^{9} \mathrm{Be},{ }^{n a t} \mathrm{C},{ }^{28} \mathrm{Si},{ }^{58} \mathrm{Ni},{ }^{62} \mathrm{Ni},{ }^{90} \mathrm{Zr},{ }^{118} \mathrm{Sn},{ }^{120} \mathrm{Sn}$, ${ }^{124} \mathrm{Sn}$, and ${ }^{154} \mathrm{Sm}$. The new spectrometer "Grand Raiden" was employed for the $\left({ }^{3} \mathrm{He}, \mathrm{t}\right)$ study for the first time. Observed $\left({ }^{3} \mathrm{He}, \mathrm{t}\right)$ spectra at $0^{\circ}$ show remarkable similarity with those from the $(p, n)$ results at intermediate energies, suggesting a simple direct reaction mechanism for the $\left({ }^{3} \mathrm{He}, \mathrm{t}\right)$ reaction at $450 \mathrm{MeV}$. The GamowTeller (GT) resonances and the spin-flip $\Delta \mathrm{L}=1$ resonances are excited very strongly. A fine structure of the GT resonances in medium-heavy nuclei is observed with an energy resolution of $210 \mathrm{keV}$. It is demonstrated that the $\left({ }^{3} \mathrm{He}, \mathrm{t}\right)$ reaction at 450 $\mathrm{MeV}$ is a powerful tool to study the spin-isospin excitations in nuclei.
\end{abstract}

\section{Introduction}

The spin-isospin excitations in nuclei have been one of the interesting subjects of experimental and theoretical investigations for a long time. Gross bumps of Ganow-Teller strengths, which were predicted long ago from the quenched $\beta$ decay strength [1], were experimentally identified in almost all nuclei by the $(p, n)$ reaction at intermediate energies $\mathrm{E} \geq 100 \mathrm{MeV}$ [2]. In the past decade, it has been shown that the charge-exchange 
reactions at small momentum transfer at intermediate energies are dominated by the strong central spin-isospin force [3].

The Gamow-Teller resonances were studied also by the alternative charge-exchange reactions like $\left({ }^{3} \mathrm{He}, \mathrm{t}\right)$ and $\left({ }^{6} \mathrm{Li},{ }^{6} \mathrm{He}\right)$ by taking advantage of high resolution and high detection efficiency. In particular, the $\left({ }^{3} \mathrm{He}, \mathrm{t}\right)$ reaction has been used to observe the GT strengths at various bombarding energies $\mathrm{E} \leq 100 \mathrm{MeV}[4,5,6,7,27,9,10]$. It is now clear that an extraction of the GT strength from the $\left({ }^{3} \mathrm{He}, \mathrm{t}\right)$ cross sections at lowbombarding energies is more difficult than for the $(p, n)$ reactions at $E \geq 100 \mathrm{MeV}$ due to the dominance of the two-step reaction mechanisms and of the non-central interaction. It has been shown experimentally that the $\left({ }^{3} \mathrm{He}, \mathrm{t}\right)$ reaction becomes a very suitable alternative to investigate the spin-isospin excitations when the bombarding energies exceed $100 \mathrm{MeV} / \mathrm{u}$ [11]. The detection of high-energy tritons from the $\left({ }^{3} \mathrm{He}, \mathrm{t}\right)$ reaction requires a spectrometer with a large magnetic rigidity. One attempt was made by the Saclay group [11] to measure the $\left({ }^{3} \mathrm{He}, \mathrm{t}\right)$ reaction at $\mathrm{E} \geq 200 \mathrm{MeV} / \mathrm{u}$. However, the energy resolution was not sufficient enough to use this reaction as a spectroscopic tool for the investigation of the details of the spin-isospin excitations in heavy nuclei.

Construction of the new high resolution spectrometer Grand Raiden [13] at the Research Center for Nuclear Physics (RCNP) has very much improved this experimental situation for the spectroscopic study. The spectrometer has two special features; One is its high momentum resolving power of $\mathrm{p} / \Delta \mathrm{p}=37,000$ and another is a large magnetic rigidity $(K=1400)$ to bend charged particles. These features make the study of the spinisospin excitations by means of the $\left({ }^{3} \mathrm{He}, \mathrm{t}\right)$ reaction very attractive. New attempts of studying the spin-isospin excitations by the $\left({ }^{3} \mathrm{He}, \mathrm{t}\right)$ reaction are now in progress taking advantage of selective excitation of the Gamow-Teller resonance at zero degree and at the high bombarding energy $E=450 \mathrm{MeV}$ with high resolution. In the present paper, we report on the new experimental data for the $\left({ }^{3} \mathrm{He}, \mathrm{t}\right)$ reaction on ${ }^{9} \mathrm{Be}$, ${ }^{n a t} \mathrm{C},{ }^{28} \mathrm{Si},{ }^{58,62} \mathrm{Ni}$, ${ }^{9} \mathrm{Zr},{ }^{118,12 n, 124} \mathrm{Sn}$, and ${ }^{154} \mathrm{Sm}$ at $\mathrm{E}=450 \mathrm{MeV}$.

\section{Experiment}

The present data were taken with a $450 \mathrm{MeV}{ }^{3} \mathrm{He}$ beam from the new $\mathrm{K}=400 \mathrm{MeV}$ ring cyclotron [12], and with a new spectrometer Grand Raiden at RCNP [13]. The 450 $\mathrm{MeV}^{3} \mathrm{He}^{++}$beam was achromatically transported from the cyclotron to the scattering chamber without any energy-defining slits in order to reduce a beam halo. The beam current on targets was about $3 \mathrm{nA}$. The targets used were metallic foils with the thicknesses of $4 \sim 7 \mathrm{mg} / \mathrm{cm}^{2}$. The spectrometer was set at $0^{\circ}$. The vertical and horizontal defining angles were, respectively, set at $\pm 20 \mathrm{mr}$.

The ${ }^{3} \mathrm{He}^{++}$beam passing through the target was introduced in the spectrometer, and was stopped in a special Faraday cup prepared at an inside wall of the vacuum chamber of the first dipole (D1) magnet. The outgoing tritons were momentum analyzed with the spectrometer and detected by the focal-plane counter system, which consisted of two 2-dimensional position-sensitive multi-wire drift chambers (MWDC) and two $\Delta \mathrm{E}$ - 
scintillation counters for particle identification [14]. The resolution was $210 \mathrm{keV}$ in FWHM. In addition to triton peaks due to the $\left({ }^{3} \mathrm{He}, \mathrm{t}\right)$ reaction, a strong peak due to ${ }^{3} \mathrm{He}^{+}$events was observed at the high-momentum side of the focal plane. The ${ }^{3} \mathrm{He}^{+}$ particles were measured simultaneously with the tritons and were used to calibrate both the energy and the scattering angles of the detected tritons. The horizontal and vertical scattering angles at the target could be determined by the ray-tracing techniques. using the information of the incidence angle of tritons at the focal plane. The obtained data at $0^{\circ}$ with the solid angle of $1.6 \mathrm{msr}$ were further divided into the data with individual narrow cones at small scattered angles in order to estimate the angular distributions at very forward angles.

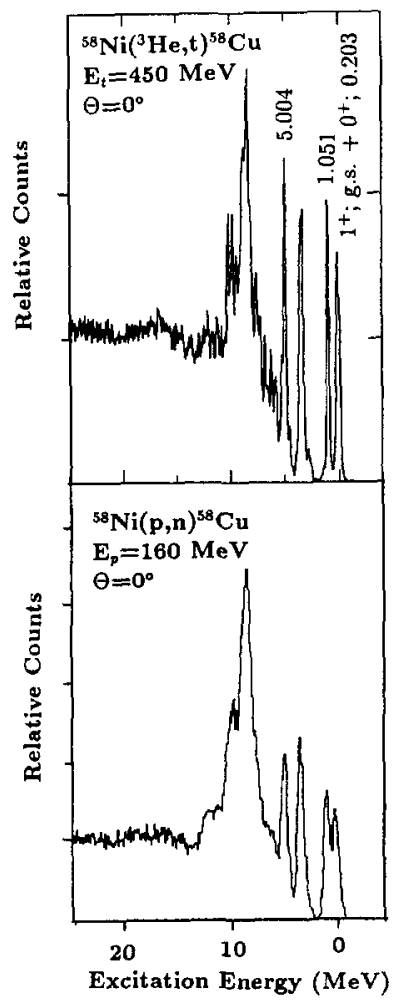

Figure 1: Comparison of the ${ }^{58} \mathrm{Ni}\left({ }^{3} \mathrm{He}, \mathrm{t}\right)$ spectrum taken at $450 \mathrm{MeV}$ with the $(\mathrm{p}, \mathrm{n})$ spectrum at $160 \mathrm{MeV}$.

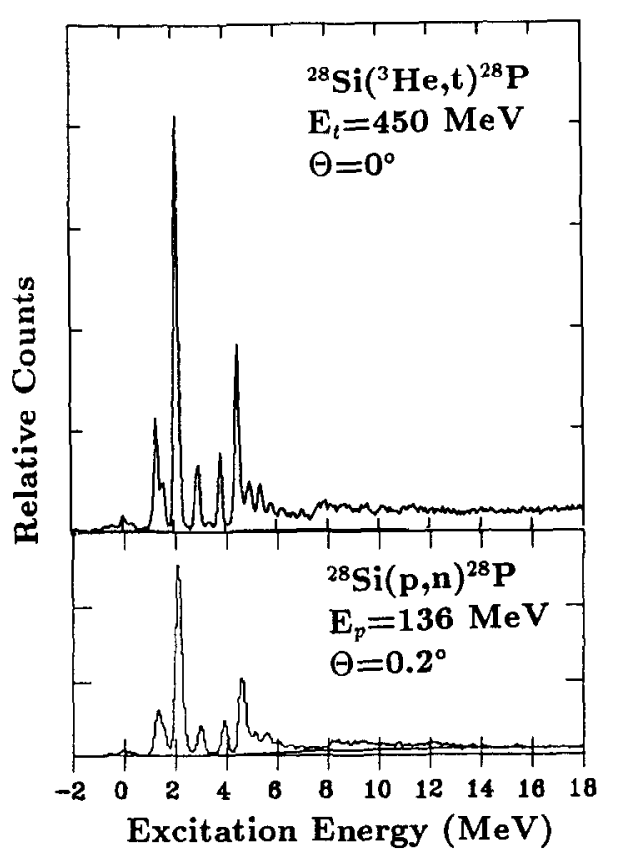

Figure 2: Comparison of the ${ }^{28} \mathrm{Si}\left({ }^{3} \mathrm{He}, \mathrm{t}\right)$ spectrum taken at $450 \mathrm{MeV}$ with the $(\mathrm{p}, \mathrm{n})$ spectrum at $136 \mathrm{MeV}$.

Figure 1 and 2 show examples of the ${ }^{58} \mathrm{Ni},{ }^{28} \mathrm{Si}\left({ }^{3} \mathrm{He}, \mathrm{t}\right)$ spectra taken at $0^{\circ}$. For comparison, the neutron spectra from the $(p, n)$ reactions taken at a bombarding energy of 160 and $136 \mathrm{MeV}[15,16]$ are shown. In a similar way to the $(\mathrm{p}, \mathrm{n})$ case, our measurement at $150 \mathrm{MeV} / \mathrm{u}$ shows a clear enhancement of excitations of the GT states. This similarity is reasonable since the ratio of spin-flip to non-spin flip contribution $\left(\mathrm{J}_{\sigma \tau} / \mathrm{J}_{\tau}\right)^{2}$ 
in the $\left({ }^{3} \mathrm{He}, \mathrm{t}\right)$ reaction is believed to be close to that in the $(\mathrm{p}, \mathrm{n})$ reaction at the same bombarding energy per nucleon [11].

\section{Discussion}

\subsection{Gamow-Teller and Fermi Transitions in the ${ }^{12,13} \mathrm{C}\left({ }^{3} \mathrm{He}, \mathrm{t}\right)^{12,13} \mathrm{~N}$ reac- tion}

The relative cross sections for the ${ }^{12,13} \mathrm{C}\left({ }^{3} \mathrm{He}, \mathrm{t}\right)$ reaction at $0^{\circ}$ have been examined by the measurement with a natural carbon target containing $1.11 \%{ }^{13} \mathrm{C}$. Taddeucci et al. [17] and Bergqvist et al. [11] have shown that the ratio of the $0^{\circ}$ cross sections in the charge exchange reaction for transitions to the ground state of ${ }^{12} \mathrm{~N}$ and to the $3.51 \mathrm{MeV}$ $3 / 2^{-}$state in ${ }^{13} \mathrm{~N}$ is a good measure to check the simplicity of the reaction mechanism. The cross section ratio $\frac{\sigma\left({ }^{12} C \rightarrow \rightarrow^{12} N(g . s .)\right)}{\sigma\left({ }^{13} C \rightarrow{ }^{13} N(3.51)\right)}$ obtained from the present experiment at $450 \mathrm{MeV}$ is shown in fig. 3 together with the ratio of $\frac{\sigma\left({ }^{13} C \rightarrow^{13} N(g . s .)\right)}{\sigma\left({ }^{13} C \rightarrow^{13} N(3.51)\right)}$. The present data at 450 $\mathrm{MeV}$ is compared with the results obtained at $200 \mathrm{MeV}$ [18] and at $\mathrm{E} \geq 200 \mathrm{MeV} / \mathrm{u}$ [11]. As shown in fig. 3 , the ratio $\frac{\left.\sigma^{12} C \rightarrow{ }^{12} N(g . s .)\right)}{\sigma\left({ }^{13} C \rightarrow^{13} N(3.51)\right)}$ is about 0.72 at $450 \mathrm{MeV}$, in agreement with the $\left({ }^{3} \mathrm{He}, \mathrm{t}\right)$ values at higher energies within uncertainties. This fact indicates that the $\left({ }^{3} \mathrm{He}, \mathrm{t}\right)$ reaction mechanism at $450 \mathrm{MeV}$ is simple similarly to the high energy case, where the single step process is predominant. It is therefore reasonable to assume that the $0^{\circ}$ cross sections are proportional to the GT $\beta$-decay strengths. At lower energies, on the other hand, this ratio decreases rapidly, suggesting that the reaction mechanism is getting more complex with decreasing the bombarding energy. The cross section ratio $\frac{\sigma\left({ }^{13} C \rightarrow{ }^{13} N(g . s .)\right)}{\sigma\left({ }^{13} C \rightarrow{ }^{13} N(3.51)\right)}$ makes it possible to estimate the close similarity between $\left({ }^{3} \mathrm{He}, \mathrm{t}\right)$ and $(\mathrm{p}, \mathrm{n})$, at least up to energies of $300 \mathrm{MeV} / \mathrm{u}$.

Figure 3: Ratios of $\frac{\sigma\left({ }^{12} C \rightarrow 12 N(g .8 .)\right)}{\sigma\left({ }^{13} C \rightarrow{ }^{13} N(3.51)\right)}$ and $\frac{\sigma\left({ }^{13} C \rightarrow^{13} N(g . s .)\right)}{\sigma\left({ }^{13} C \rightarrow{ }^{13} N(3.51)\right)}$. The lines are drawn to guide the eye.

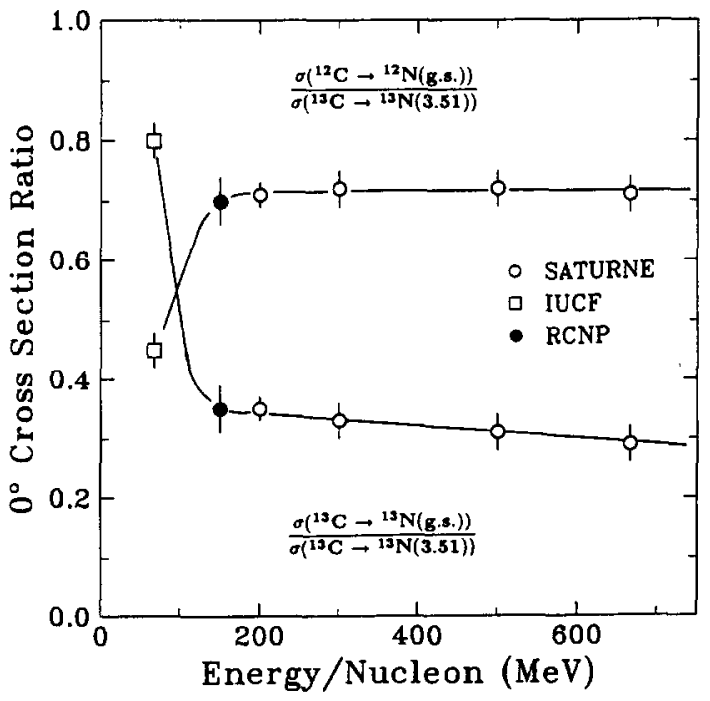




\subsection{Excitation of the GT and spin-flip $\Delta \mathrm{L}=1$ resonances}

Figure 4 shows the $\left({ }^{3} \mathrm{He}, \mathrm{t}\right)$ spectra taken at $0^{\circ}$ for targets of ${ }^{62} \mathrm{Ni},{ }^{90} \mathrm{Zr},{ }^{118} \mathrm{Sn},{ }^{120} \mathrm{Sn}$, ${ }^{124} \mathrm{Sn}$, and ${ }^{154} \mathrm{Sm}$. Our measurement at $150 \mathrm{MeV} / \mathrm{u}$ shows a clear enhancement of the GT resonance compatible with the $(p, n)$ reactions at intermediate energies. The broad bumps superposed on the continuum around $\mathrm{E}_{x}=20 \mathrm{MeV}$ is due to the excitation of the spin-flip dipole $\Delta \mathrm{L}=1$ resonances (SDR) which have been suggested from the retarded first-forbidden $\beta$ transitions mediated by the operator $\mathrm{r}\left[\mathrm{Y}_{1} \times \sigma\right] \tau$ [19]. By making a "true" $0^{\circ}$ spectrum gated on the narrow cone around $0^{\circ}$ by ray-tracing techniques, we have confirmed that the SDR bump is less pronounced and is really not forward peaked.
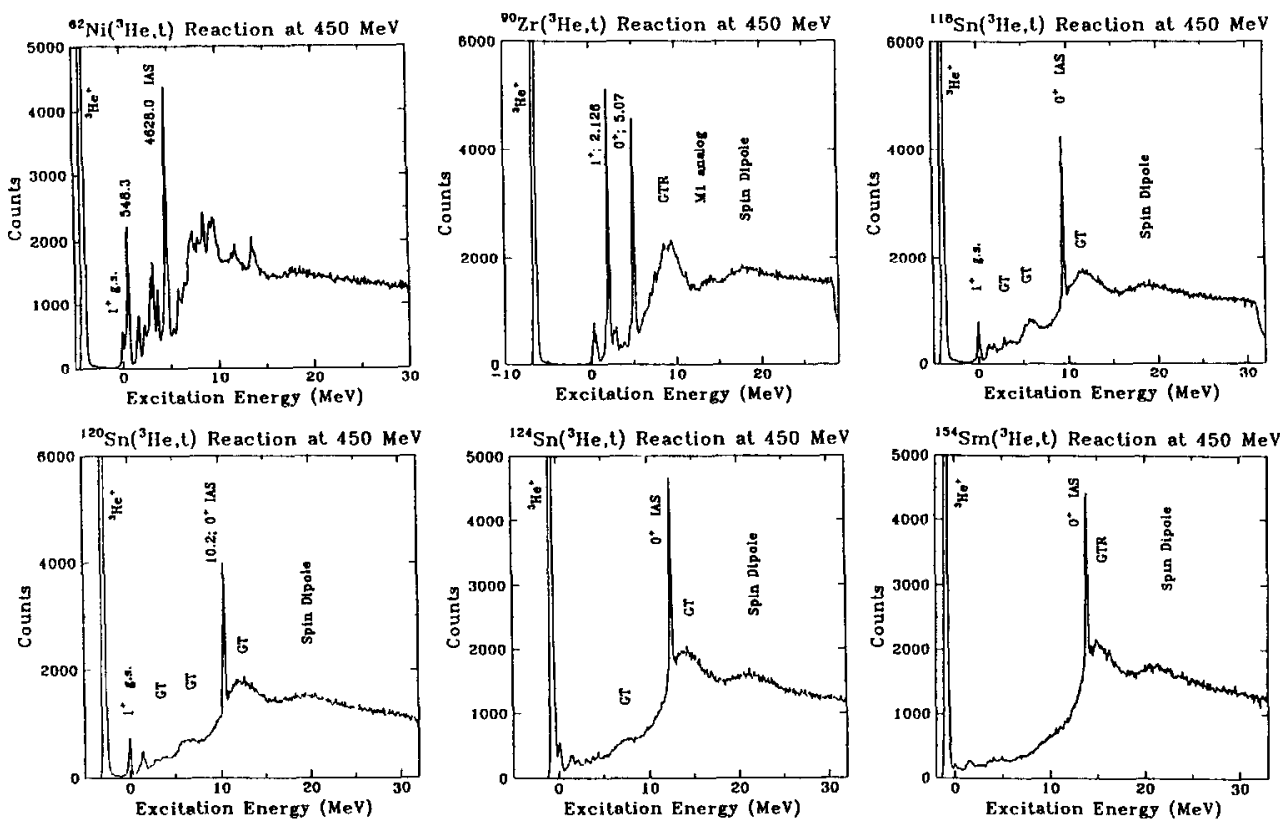

Figure 4: Singles $\left({ }^{3} \mathrm{He}, \mathrm{t}\right)$ spectra obtained for ${ }^{62} \mathrm{Ni},{ }^{90} \mathrm{Zr},{ }^{118,120,124} \mathrm{Sn}$ and ${ }^{154} \mathrm{Sm}$ at $\mathrm{E}=450 \mathrm{MeV}$ at $0^{\circ}$ with the spectrometer Grand Raiden. Spectra were obtained with an opening angle of $\pm 20 \mathrm{mrad}$ both vertically and horizontally.

From the cross section ratio and the known $\mathrm{B}(\mathrm{GT}) / \mathrm{B}(\mathrm{F})$ ratio for the $2.126 \mathrm{MeV}$ $1^{+}$state and the $5.07 \mathrm{MeV}$ IAS in ${ }^{90} \mathrm{Zr}$, one can estimate the ratio

$$
\epsilon=\left(\frac{J_{\sigma \tau}}{J_{\tau}}\right)^{2} \frac{N_{\sigma \tau}}{N_{\tau}} \approx\left(\frac{\sigma_{G T}\left(0^{\circ}\right)}{\sigma_{F}\left(0^{\circ}\right)}\right) \frac{B(F)}{B(G T)} \sim 6.4,
$$

where $\mathrm{N}_{\sigma \tau}$ and $\mathrm{N}_{\tau}$ are the distortion factors, and the $\mathrm{Q}$ dependence of the transferred momentum is neglected. A similar value of $\epsilon=6.6$ is obtained for the ${ }^{58} \mathrm{Ni}\left({ }^{3} \mathrm{He}, \mathrm{t}\right)$ reac- 
tion at $450 \mathrm{MeV}$. If one assumes $\mathrm{N}_{\sigma \tau} / \mathrm{N}_{\tau} \sim 1$, the ratio $\mathrm{J}_{\sigma \tau} / \mathrm{J}_{\tau}$ agrees with the results calculated by Franey and Love [20] at $150 \mathrm{MeV}$.

It is very surprising that when the GT resonance in ${ }^{90} \mathrm{Nb}$ is observed under the condition of the experimental resolution of $210 \mathrm{keV}$, the resonance seems to be an ensemble of many discrete $1^{+}$levels. It would be very interesting to examine the reason why this kind of fine structure still appears for the GT resonance at the highly excited energy region in the medium heavy nuclei like ${ }^{90} \mathrm{Nb}$ where the extremely high level density of $1^{+}$states is expected. In addition, it should be noted that we clearly see the bump of the $\mathrm{M} 1$ analog resonance of ${ }^{90} \mathrm{Zr}$ with $\mathrm{T}=5$ at $\mathrm{E}_{x}=13 \sim 15 \mathrm{MeV}[21,22]$.

Jänecke et al. [23] report that the giant GT resonances in ${ }^{118,120,124} \mathrm{Sb}$ isotopes are fragmented into three (or more) components corresponding to the direct-, corepolarization and back-spin-flip GT strengths. Our observation at $450 \mathrm{MeV}$ is consistent to their results obtained in the $\left({ }^{3} \mathrm{He}, \mathrm{t}\right)$ measurements at $200 \mathrm{MeV}$.

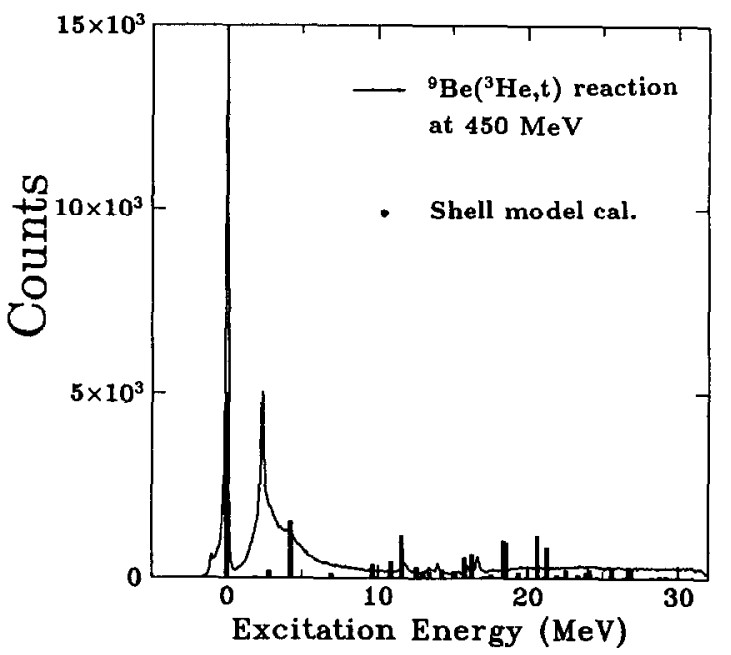

The giant electric dipole resonance in deformed nuclei is known to split into two components, representing the existence of two types of oscillations in the direction of the symmetry axis and in the perpendicular direction. An analogous splitting is expected for the spin-flip $\Delta \mathrm{L}=1$ resonance in ${ }^{154} \mathrm{Sm}$. As a consequence, the width of the SDR in ${ }^{154} \mathrm{Sm}$ should be observed to be broader than those in the $\mathrm{Sn}$ isotopes. It is, however, difficult to judge from the present experiment whether the broad bump at $E_{x}=9 \sim 25$ $\mathrm{MeV}$ separates into two components or is broader. Further experimental efforts may be necessary to separate the SDR from the continuum. It is a very interesting theoretical subject to predict the shape of the SDR in deformed nuclei.

The ${ }^{9} \mathrm{Be}\left({ }^{3} \mathrm{He}, \mathrm{t}\right){ }^{9} \mathrm{~B}$ spectrum shows a characteristic feature. The transitions to the 3/2- 0.0 MeV-, 5/2- 2.36 MeV-, 2.79 MeV-, 4.8 MeV-, $11.78 \mathrm{MeV}-$, and $14.01 \mathrm{MeV}-$ states are observed. Almost all $\Delta \mathrm{L}=0$ transitions are concentrated in the region below $\mathrm{E}_{x}=10 \mathrm{MeV}$ as is shown in Figure 5, where the experimental data are compared with the 
results of a shell model calculation for the $\Delta \mathrm{L}=0$ transitions mediated by the operators $\sigma \tau$ and $\tau$ [24]. The shell model calculation predicts the major strength of spin-isospin $\Delta \mathrm{L}=0$ transitions at energies above $10 \mathrm{MeV}$ but it cannot reproduce the strong concentration of the $\Delta \mathrm{L}=0$ transitions below $10 \mathrm{MeV}$. This general trend in shell-model calculations does not depend on the choice of the effective interactions [25] for the pshell nuclei. Ohtsubo [24] pointcd out that this concentration could be qualitatively understood by taking into accounts the cluster-model wave function with the $\alpha$-N- $\alpha$ structure both for ${ }^{9} \mathrm{Be}$ and ${ }^{9} \mathrm{~B}$.

\subsection{Fine structure of the GT strength in ${ }^{58} \mathrm{Cu}$}

Because of the good resolution of the present ${ }^{58} \mathrm{Ni}\left({ }^{3} \mathrm{He}, \mathrm{t}\right)$ experiment $(210 \mathrm{keV}$ in FWHM), we observed many discrete peaks up to $\mathrm{E}_{x}=15 \mathrm{MeV}$. In particular, the bump at $\mathrm{E}_{x}=6 \sim 12 \mathrm{MeV}$ is resolved into many distinct peaks, which were unresolved in the $(\mathrm{p}, \mathrm{n})$ experiment at intermediate energies.

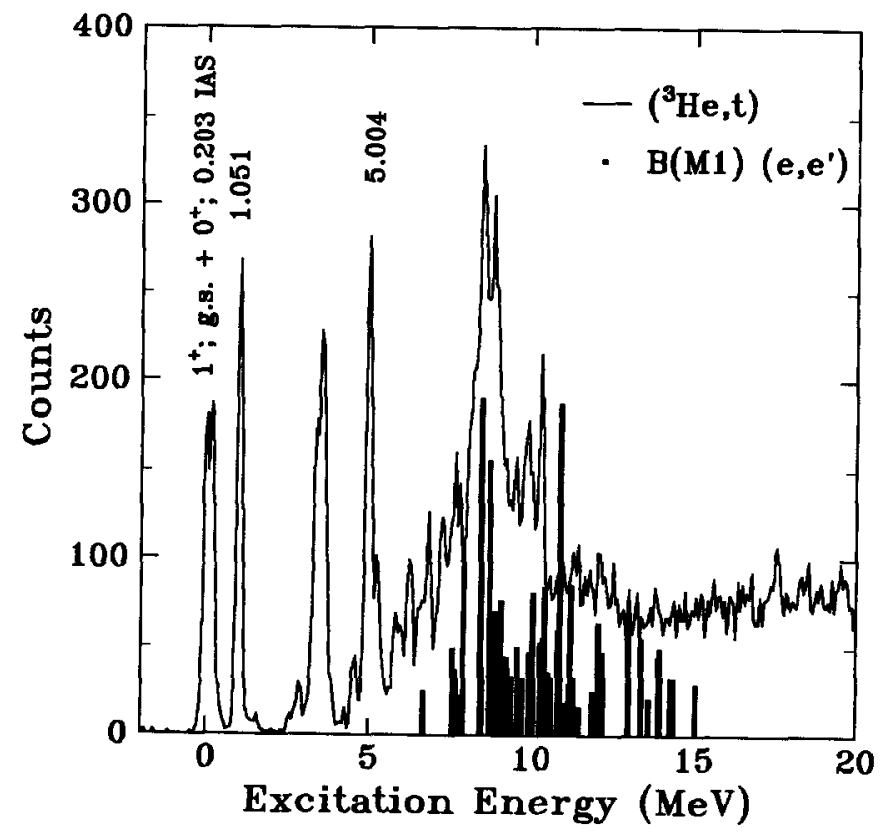

Figure 6: ${ }^{58} \mathrm{Ni}\left({ }^{3} \mathrm{He}, \mathrm{t}\right)^{58} \mathrm{Cu}$ spectrum obtained with a narrow software gate on the scattering angles of $\pm 5 \mathrm{mr}$, both in horizontal and vertical directions. The M1 strength distribution obtained from (e,e') data is displayed with the energy off-set of $0.203 \mathrm{MeV}$.

The GT strength distribution measured with the charge-exchange reactions are re- 
lated to the M1 strength distributions. Electromagnetic (e.m.) M1 excitations are induced both by the spin and orbital magnetization interactions. The spin and orbital parts in the e.m. M1 excitation interfere either constructively or destructively with each other. Furthermore, the contribution of the backward transitions is always destructive in the case of the M1 transitions. Although there is no precise correlation between the M1 strength and GT strength for each individual state, we can, however, still expect a one-to-one correspondence between the M1 and GT states, which should be clarified on the basis of their isospin.

In Fig. 6, the $\left({ }^{3} \mathrm{He}, \mathrm{t}\right)$ cross sections are compared to the M1 strength distribution from (e,e') [26]. One can remark that we have observed analogous transitions in the two spectra, especially in the region of $\mathrm{E}_{x} \sim 8 \mathrm{MeV}$, where the $\mathrm{T}=1,1^{+}$states are expected. The B(M1) strength distributions has a shape similar to the observed $0^{\circ}\left({ }^{3} \mathrm{He}, \mathrm{t}\right)$ cross sections. This indicates that the main part of the bump at $8 \mathrm{MeV}$ consists of many $1^{+}$ states with $T=1$. The detailed difference between the $M 1$ and GT strength distributions would come from the effect of the orbital excitation contribution in M1 excitations.

The $\mathrm{T}=2$ isobaric analog states of the $\mathrm{T}=2, \mathrm{M} 1$ states in ${ }^{58} \mathrm{Ni}$ are hindered in chargeexchange reactions by a factor of $\frac{1}{2 T_{0}+1}=1 / 3$. Thus they are not expected to be excited strongly in the $\left({ }^{3} \mathrm{He}, \mathrm{t}\right)$ spectrum at $0^{\circ}$. In fact, the isobaric analog state of the strong $1^{+}$state at $10.67 \mathrm{MeV}$ in ${ }^{58} \mathrm{Ni}$ is only weakly excited in the present experiment, which actually suggests that this state has isospin $T=2$.

\subsection{Proton decay from the spin-flip dipole resonance in ${ }^{12} \mathrm{~N}$}

Microscopic structure of the giant resonances in nuclei can be well studied by measuring the decay particles. Especially, proton decay measurement from the GTR and SDR is important since we can infer the wave function of the resonance through the decay pattern and its decay strength to the final discrete levels. One of the good examples is the measurement of the proton decay from the spin-flip dipole resonance with $\mathrm{J}^{\pi}=0^{-}$, $1^{-}$and $2^{-}$in ${ }^{12} \mathrm{~N}$, which are excited via the $\left({ }^{3} \mathrm{He}, \mathrm{t}\right)$ or $(\mathrm{p}, \mathrm{n})$ reactions. The existence of the $1^{-}$and $2^{-}$states is well established experimentally. However, there is no clear experimental evidence for the $0^{-}$state whose strength is predicted at the location slightly higher than the $1^{-}$state $[27,28]$.

Coincidence measurements of the ${ }^{12} \mathrm{C}\left({ }^{3} \mathrm{He}, \mathrm{tp}\right){ }^{11} \mathrm{C}$ reaction (see also [29]) has been made with the new spectrometer Grand Raiden by utilizing the advantages; 1) tritons can be detected at $0^{\circ}, 2$ ) the incoming ${ }^{3} \mathrm{He}$ beam is stopped at the inside wall of the D1 magnet where it is far from the target, 3) the ${ }^{3} \mathrm{He}$ beam without a halo can be transported in an achromatic mode to the target from the RCNP ring cyclotron, 4) the $\left({ }^{3} \mathrm{He}, \mathrm{t}\right)$ reaction at $450 \mathrm{MeV}$ preferentially excites spin-flip states. Preliminary data for the coincidence measurement to search for the $0^{-}$state in ${ }^{12} \mathrm{C}$ are shown in Fig. 7. One can clearly see the loci corresponding to the decay proton events to the final state in ${ }^{11} \mathrm{C}$, indicating the successful trial of the difficult coincidence measurement at intermediate energies. Detailed analyses of the obtained data is now in progress. We expect that our new data will furnish an evidence for the $0^{-}$state and new information 

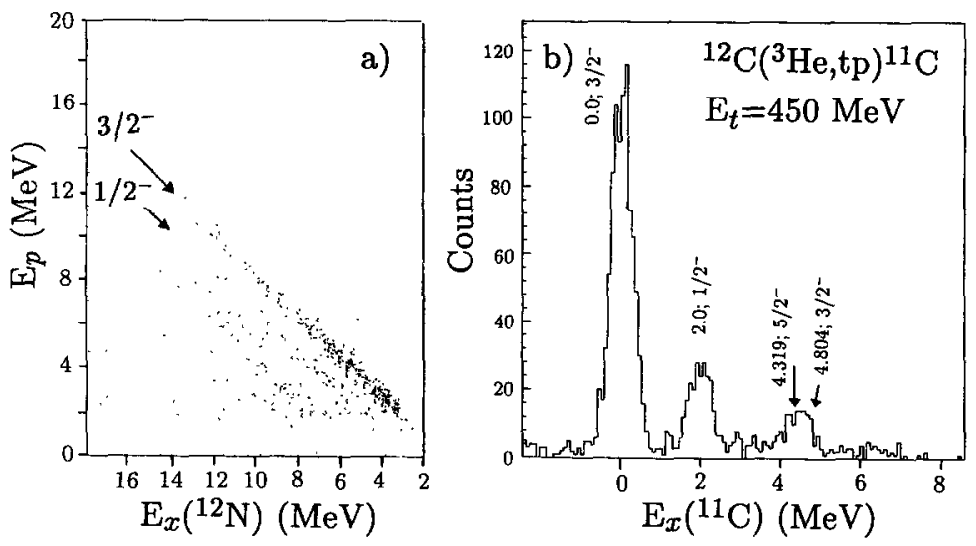

Figure 7: (a) Two-dimensional scatter plot of coincidence events for decay-proton and tritons. The loci indicate proton decay to low-lying final states in ${ }^{11} \mathrm{C}$. (b) Corresponding total kinetic energy spectrum.

on the microscopic structure of the spin flip dipole resonance in ${ }^{12} \mathrm{C}$.

\section{Conclusions}

The $\left({ }^{3} \mathrm{He}, \mathrm{t}\right)$ reaction has been measured at $450 \mathrm{MeV}$ at $0^{\circ}$ on several target nuclei. Strong selectivity for spin-isospin excitations has been shown. It is found that the reaction mechanism of the $\left({ }^{3} \mathrm{Hc}, \mathrm{t}\right)$ reaction at $450 \mathrm{McV}$ is simple, compatible with the $(p, n)$ reaction at intermediate energies $\mathrm{E} \geq 100 \mathrm{MeV}$. This shows that the high-resolution $\left({ }^{3} \mathrm{He}, \mathrm{t}\right)$ measurements at $450 \mathrm{MeV}$ can be a very useful tool in mapping the detailed GT transition strengths in nuclei. In addition to the singles high-resolution $\left({ }^{3} \mathrm{He}, \mathrm{t}\right)$ measurements, coincidence measurements with decay particles following the excitation of the GTR and SDR by the $\left({ }^{3} \mathrm{He}, \mathrm{t}\right)$ reaction are one of the best suitable experiments for the spectrometer Grand Raiden, which have not been possible to be performed with the $(\mathrm{p}, \mathrm{n})$ reaction.

The authors wish to thank the staff of RCNP, for their enthusiastic effort to obtain a high quality ${ }^{3} \mathrm{He}$ beam without a halo from the new ring cyclotron. One of the authors (M.F.) would like to acknowledge Prof. Ohtsubo for calculating the GT and Fermi transitions from ${ }^{9} \mathrm{Be}$ in the shell-model frame work. This experiment was performed at RCNP under the Program Number E34. Two of the authors (M.N.H. and J.J.) acknowledge the JSPS fellowships allowing them to participate in this experiment. 


\section{References}

[1] K.I. Ikeda, S. Fujii and J.I. Fujita, Phys. Lett. 3 (1963) 271; J.I. Fujita and K.I. Ikeda, Nucl. Phys. 67 (1965) 145.

[2] C.D. Goodman et al., Phys. Rev. Lett. 44 (1980) 1755.

[3] F. Osterfeld, Rev. Mod. Phys. 64 (1992) 491.

[4] A. Galonsky et al., Phys. Rev. Lett. 45 (1978) 176.

[5] D. Ovazza et al., Phys. Rev. C18 (1978) 2438.

[6] C. Gaarde et al., Nucl. Phys. A334 (1980) 248; C. Gaarde et al., Nucl. Phys. A346 (1980) 497.

[7] S.L. Tabor et al., Nucl. Phys. A422 (1984) 12.

[8] W.A. Sterrenburg et al., Nucl. Phys. A405 (1983) 109.

[9] S.Y. Van der Werf et al., Nucl. Phys. A496 (1089) 305.

[10] J. Jänecke et al., Nucl. Phys. A526 (1991) 1.

[11] I. Bergqvist et al., Nucl. Phys. A469 (1987) 648.

[12] I. Miura et al., RCNP Annual Report (1991) pp. 149.

[13] M. Fujiwara et al., Nucl. Instr. Meth. Nucl. Res. A, to be published;M. Fujiwara, in Proc. 5th French-Japanese Symposium on Nuclear Physics, Dogashima, Japan, (September 26-30, 1989 ) pp. 348.

[14] T. Noro et al., RCNP Annual Report (1991) pp. 177.

[15] J. Rapaport et al., Nucl. Phys. A410 (1983) 371.

[16] B.A. Anderson et al., Phys. Rev. C43 (1991) 50.

[17] T.N. Taddeucci et al., Nucl. Phys. A469 (1987) 125.

[18] J. Jänecke, private communication.

[19] H. Ejiri, K. Ikeda and J.I. Fujita, Phys. Rev. 176 (1968) 1277; H. Ejiri and J.I. Fujita., Phys. Rep. 38C (1978) 85.

[20] M.A. Franey and W.G. Love, Phys. Rev. C31 (1985) 488.

[21] D.E. Bainum et al., Phys. Rev. Lett. 44 (1980) 1751.

[22] N. Anantaraman et al., Phys. Rev. Lett. 46 (1981) 1318.

[23] J. Jänecke et al., to be published in Nucl. Phys. A.

[24] H. Ohtsubo, private communication.

[25] S. Cohen and D. Kurath, Nucl. Phys. 73 (1965) 1.

[26] W. Mettner et al., Nucl. Phys. A473 (1987) 160.

[27] W.A. Sterrenburg et al., Nucl. Phys. A405 (1983) 109.

[28] M.B. Greenfield et al., Nucl. Phys. A524 (1991) 228.

[29] Talk by J. Jänecke; oral presentation in this conference on the ${ }^{208} \mathrm{~Pb}\left({ }^{3} \mathrm{He}, \mathrm{tp}\right)^{207} \mathrm{~Pb}$ experiment. 